\title{
NEWS ON BEAM DYNAMICS AT SUPER-ACO*
}

\author{
$\underline{\text { P. Brunelle }}^{\#}$, J.-C. Besson, L. Cassinari, J.-M. Cousin, J. Darpentigny, G. Flynn, F. Girault, \\ J.-F. Lamarre, A. Nadji, B. Rieul, M. Sommer \\ LURE, Université Paris-Sud, Bât. 209A, BP 34, 91898 Orsay Cedex, France
}

\begin{abstract}
The $0.8 \mathrm{GeV}$ storage ring Super-ACO is now fully equipped with undulators. Two new undulators have been recently installed. Their effects on beam dynamics have been studied. In addition, new developments are continuously in progress in order to optimize the beam performances for the users. A beam position feedback system is now operational. A $500 \mathrm{MHz}$ RF cavity allows to reduce the bunch length and increase the FEL gain. A feedback system on betatron tunes has been tested using a very precise detection. Some experiments on beam dynamics have been performed in order to understand and improve beam lifetime. The variation of tunes with momentum has been measured, for several configurations of undulators (up to 6 undulators closed), and compared with computed results.
\end{abstract}

\section{INTRODUCTION}

Since three years, the LURE machine group has developed new equipments for the Super-ACO storage ring. A $500 \mathrm{MHz}$ cavity is now operational and used for Free Electron Laser (FEL) operation in addition to the $100 \mathrm{MHz}$ cavity. Two undulators have been installed in the two last free straight sections of the ring. A feedback system on beam position is fully tested and will be used routinely this year. A feedback system on horizontal and vertical tunes has been developed in order to compensate for tune variation introduced by field variations in undulators. In parallel, studies on beam dynamics have been performed in order to evaluate undulator field tolerances and to improve the optical model of the machine.

\section{THE 500 MHz HARMONIC CAVITY}

A new active $500 \mathrm{MHz}$ fifth harmonic RF cavity was installed on Super-ACO in January 1997 primarily for the purpose of reducing bunch length for FEL and timeresolved synchrotron radiation experiments [1]. To date bunch length reduction factors up to 3.5 have been obtained but many single-bunch coherent beam instability phenomena are observed with very short bunches [2]. Nevertheless, significant improvements in FEL performance have been obtained [3].

\footnotetext{
*Work supported by CNRS, CEA and MENESR.

\# Email : brunelle@lure.u-psud.fr.
}

In the future we will attempt to operate the harmonic RF system at higher power levels $(25 \mathrm{~kW}$ rather than $14 \mathrm{~kW}$ currently) and observe the effect on beam stability and bunch length. In addition we will attempt to use the cavity in a bunch-lengthening mode in the hope of improving beam lifetime in normal operation. This will require operating the cavity at very low field levels (roughly $1 / 5$ of the principal RF voltage) of about $34 \mathrm{kV}$. In this configuration a direct RF feedback system will be needed to avoid type II Robinson instability. Tests of such a system are currently underway both on the principal and harmonic RF systems.

\section{INSERTION DEVICES}

The two last undulators installed on Super-ACO are SU8 (a planar symetric hybrid undulator made by Danfysik) and OPHéLIE (an electromagnetic crossed overlapped undulator). The design, construction and commissioning of OPHéLIE are described in [4]. The SU8 characteristics are $B \max =0.48 \mathrm{~T}, \mathrm{~N} \times \lambda=$ $21.5 \times 100 \mathrm{~mm}$ with a minimum gap of $39 \mathrm{~mm}$. This undulator has no field integral default at any gaps. The transverse peak field homogeneity was limited to $50 \mathrm{~T} . \mathrm{m}^{-2}$ in order to avoid strong non linear effects. An automatic global compensation of its strong focusing effect $\left(\Delta v_{z \max }=2.810^{-2}\right)$ is made at each gap using two quadrupole families. An increase of the vertical chromaticity, due to the beta-function beat, was measured at minimum gap, with a $10 \%$ reduction of the beam lifetime [5].

\section{BEAM POSITION FEEDBACK}

A fast global feedback system is used on Super-ACO in order to improve beam stability [6]. The feedback uses all the 16 machine BPMs and 8 correctors in each plane to correct the orbit at a $16 \mathrm{kHz}$ rate. The goal is to provide a correction from DC to $100 \mathrm{~Hz}$, both in horizontal (x) and vertical (z) plane. The BPM signal processors are Bergoz developed heterodyne receivers working at $200 \mathrm{MHz}$ (twice the RF frequency) with a resolution of $0.5 \mu \mathrm{m} / \sqrt{\mathrm{Hz}}$. The fast corrections are computed by 8 DSP boards which acquire the $\mathrm{x}$ and $\mathrm{z}$ signals from the $16 \mathrm{BPM}$ processors (2 BPMs per DSP). The 8 DSP boards are linked via a fast high rate digital serial link and form a ring controlled by a DSP master board. It takes $60 \mu$ s to complete the whole acquisition, transmission, checking and calculation cycle for the 32 data (16 $\mathrm{x}$ and $16 \mathrm{z}$ ). 
The correction kicks are produced by 8 coils in each plane, located inside quadrupole magnets. They are powered by current-controlled wideband power amplifiers.

Two correction algorithms have been tested, a harmonic based method, and a direct inversion of the response matrix using the SVD method.

The achieved bandwith of the feedback is $40 \mathrm{~Hz}$ in the horizontal plane, and $150 \mathrm{~Hz}$ in the vertical plane. Additional tests are being carried out in order to have the feedback operational during user sessions.

\section{BETATRON TUNE FEEDBACK}

Slow Feedback : This system uses a data processing microcontroller to analyze the video signal output of a spectrum analyzer. The feedback is made by dialog with the quadrupole power supply control system [7] (Fig. 1). The correction is made every $1.2 \mathrm{~s}$ with a resolution of $\pm 110^{-4}$. A typical response is shown in figure 2 where the feedback compensates the tune shift due to the gap variation of the SU3 undulator of Super-ACO.

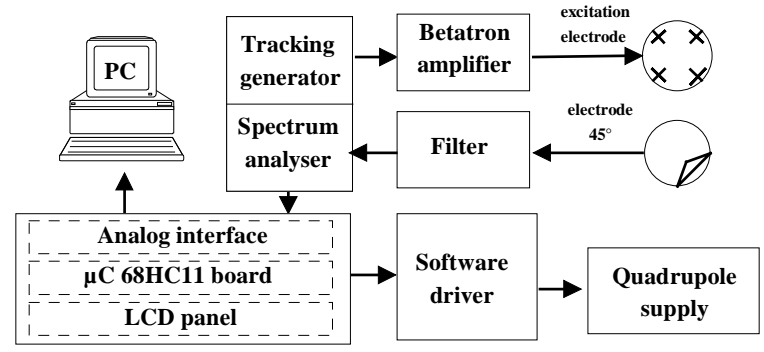

Fig. 1. Slow feedback system simplified schematic.

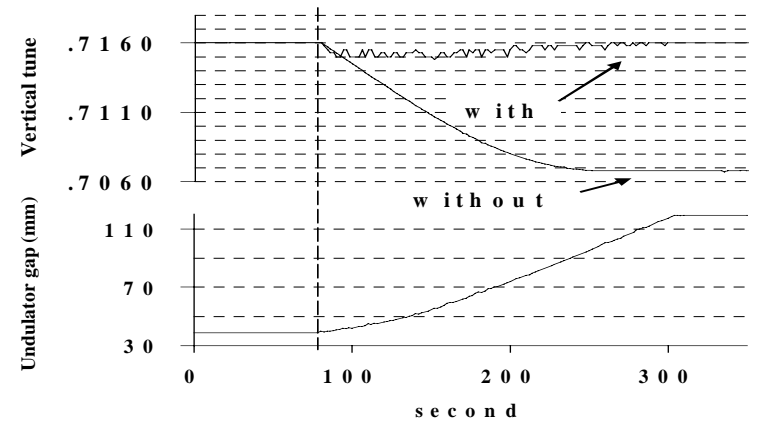

Fig. 2. Slow feedback system typical response.

Fast feedback : Two fast feedback systems are currently being developed. The first is based on digital analysis of the signal using a DSP while the second uses a phase locked loop.

Digital system (DSP) [8] : A TMS320C6201 DSP is used to determine the betatron tunes by FHT of a beam electrode signal. A tune resolution of $10^{-4}(416 \mathrm{~Hz})$ is obtained by acquiring 32000 samples. With a processing power of 1600 Mips the DSP computes a spectrum every $10 \mathrm{~ms}$, with the possibility of speeding up to every $1 \mathrm{~ms}$ (Fig. 3). This system is able to operate with low stored beam current or with a low excitation level.

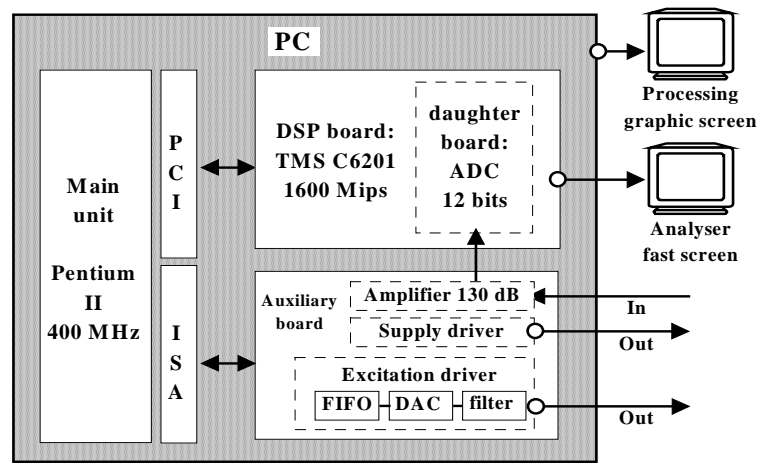

Fig. 3. Fast digital feedback system simplified schematic.

Analog system (PLL): The measurement presented here uses an analogy with a resonant electronic circuit (Fig. 4).

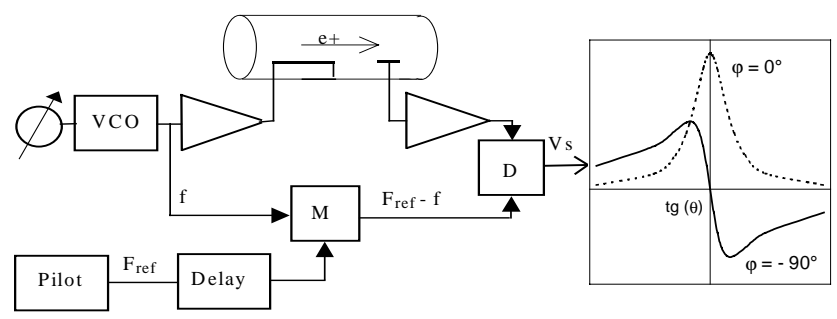

Fig. 4. Fast analog feedback system simplified schematic.

The signal delivered by the generator (VCO) at the frequency $f$ is sent simultaneously to the beam excitation amplifier and to a mixer (M) which transposes this frequency to the value $F_{r e f}-f$. A synchronous demodulator (D) compares this voltage to the detected signal and delivers a signal proportional to $V_{s}=\cos \theta \cos [\phi-\theta]$. In this expression $\theta$ represents the difference in phase with regard to the resonance and $\phi$ is given by the sum of the phase shifts and delays of the various loop elements. If $\phi=-90^{\circ}$ and is independant of $f$ one obtains a phase locked loop by using the output voltage, $V_{s}$ to control the generator frequency. The generator is then locked to the betatron frequency. On DCI [9] the precision obtained is $10^{-4}$ and the response time is $1 \mathrm{~ms}$. The system currently under development acts on the two betatron tunes independantly and is driven by a microcontroller. The tune information obtained from this system in either digital or analog form will be used to directly control the storage ring magnetic element power supplies thus providing the feedback. 


\section{TUNE VARIATION WITH MOMENTUM}

On Super-ACO, for large momentum deviations $(\mathrm{dp} / \mathrm{p}>$ $0.3 \%$ ), tune variations are no longer linear and take into account non linear effects due to higher order field components and undulator fields. The aim of this study was double: compare the tune variation computed with the BETA code [10] to the one measured on the ring when undulators are all open, and measure the effect of undulators in order to understand energy acceptance limitation. The experimental tune variation [11] was measured using RF frequency variations up to $\pm 30 \mathrm{kHz}$ $(\mathrm{dp} / \mathrm{p}= \pm 2 \%)$. The higher order field components of magnetic elements have been deduced from magnetic measurements and introduced in the BETA code as thin lenses. Figure 5 shows that the maximum stable $\mathrm{dp} / \mathrm{p}$ values are larger than the RF energy acceptance $( \pm 1.8 \%)$. The computed $v_{\mathrm{x}}$ variation is rather different from the experimental one for $\mathrm{dp} / \mathrm{p}>0$ while the agreement is quite good for $v_{z}$ variation. A good agreement for $v_{x}$ can be obtained by adjusting the decapolar component generated by sextupoles.

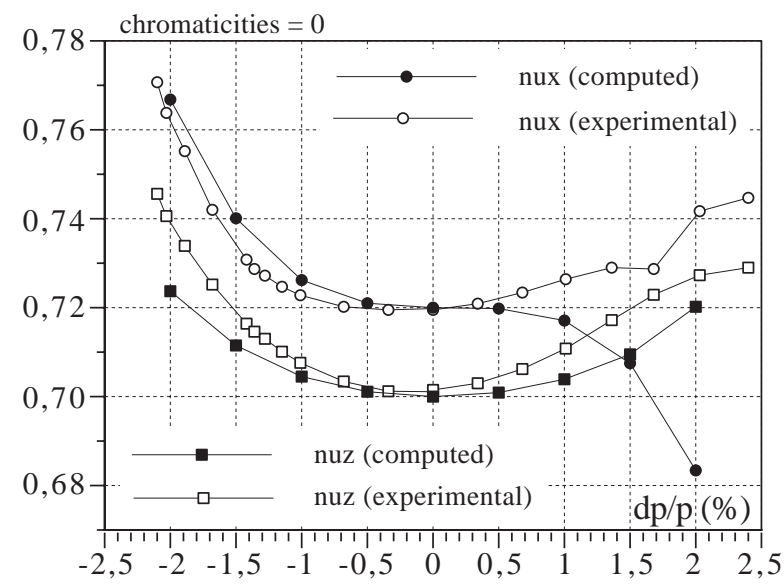

Fig. 5. Tune variation with momentum (open undulators).

Figure 6 shows the measured effect of the first four undulators (SU2, SU3, SU6, SU7) closed at nominal gap, in the positive chromaticity operation mode. The non linearities occur essentially in the vertical plane (undulator field plane) and reduce the maximum stable $\mathrm{dp} / \mathrm{p}$ values (beam losses have been observed near the $3 v_{z}=5$ resonance). The measurements have been performed for two optics with the same tunes : a 4 fold symmetry optic and a quasi 8 fold symmetry obtained with equal $\beta$ functions in the 8 straight sections. The symmetrization reduces the undulator effect and leads to an increase of beam lifetime because the maximum stable $\mathrm{dp} / \mathrm{p}$ values are larger. Tune variation versus momentum introduced by the two last undulators (SU5 and SU8) was found to be negligible.
Other tests have shown that an increase of $v_{z}$ leads to a beam lifetime reduction when the Touschek effect is dominant. In fact, when $v_{\mathrm{z}}$ is increased, the effect of the $3 v_{z}=5$ resonance does not occur any more for large negative $\mathrm{dp} / \mathrm{p}$ values. But the $4 v_{\mathrm{z}}=7$ resonance, excited by undulators, becomes a limitation for positive $\mathrm{dp} / \mathrm{p}$ values.

All these experimental results show the influence of the tune variation with momentum in the Touschek lifetime behaviour.

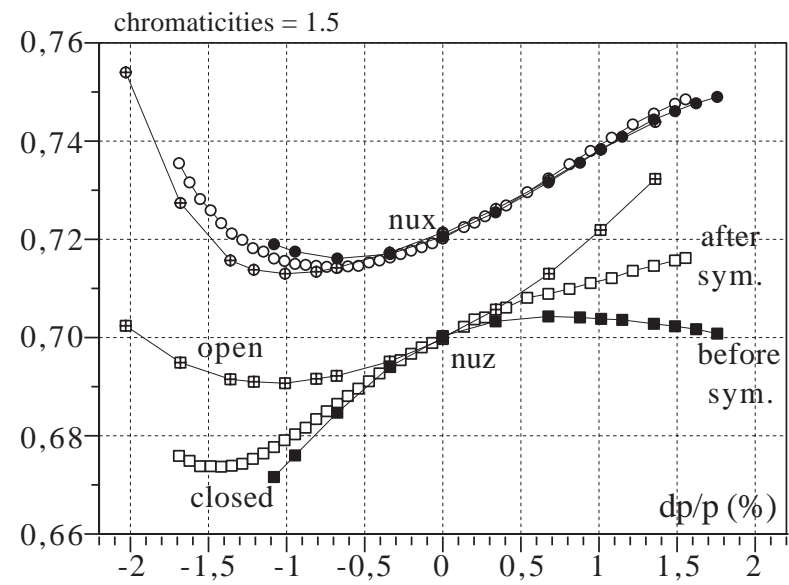

Fig. 6. Effect of undulators.

\section{ACKNOWLEDGEMENTS}

It is a pleasure to thank all the members of the technical and operation staff of Super-ACO for their collaboration.

\section{REFERENCES}

[1] G. Flynn et al., EPAC 98, pp. 1767-1769.

[2] G. Flynn et al., EPAC 98, pp. 954-956.

[3] D. Nutarelli et al., EPAC 98, pp. 670-672.

[4] M. Corlier et al., this conference.

[5] P. Brunelle, internal report, Super-ACO/97-05.

[6] L. Cassinari et al., this conference.

[7] J.-M. Cousin et al., internal reports, RT/99-01, Super-ACO/99-03.

[8] J.-M. Cousin et al., internal report, RT/99-03.

[9] J.-F. Lamarre and H. Zyngier, inter. rep. NI/96-21.

[10] J. Payet, BETA-LNS code.

[11] P. Brunelle, internal report, Super-ACO/99-04. 